\title{
Time to Take Actions to Reduce the Harmful Use of Alcohol in China
}

\author{
Ning Ji'; Yamin Bai ${ }^{1, *}$; Jianwei $\mathrm{Xu}^{1}$; Min Liu ${ }^{1}$; Ainan Jia ${ }^{1}$
}

Harmful use of alcohol negatively impacts and harms the drinkers, the people around them, and society at large. Harmful use of alcohol ranks among the top 5 risk factors for disease, disability, and death worldwide, and alcohol consumption is related to approximately 230 health conditions including infectious disease, noncommunicable diseases, and injuries (1-2).

\section{DISEASE BURDEN OF ALCOHOL}

Worldwide, alcohol use was responsible for some 3 million deaths (5.3\% of all deaths) and 132.6 million disability-adjusted life years (DALYs) $(5.1 \%$ of all DALYs) in 2016 (2). In China, alcohol caused 381,200 deaths and decreased people's life expectancy by an average of 0.43 years in 2013 (3). In that year, in terms of population attributable risk of deaths, $44.56 \%$ of cirrhosis, $28.80 \%$ of esophageal cancer, $27.65 \%$ of nasopharyngeal cancer, $27.45 \%$ of epilepsy, and $25.13 \%$ of liver cancer were attributable to alcohol consumption in China (3). Disturbingly, the burden of disease and injuries resulting from alcohol consumption increased rapidly in China. According to the global burden of disease (GBD) study, from 1990 to 2017, the total number of DALYs and the agestandardized DALY rate due to alcohol consumption increased by $58.51 \%$ and $16.02 \%$, respectively (4).

\section{ALCOHOL CONSUMPTION}

Alcohol consumption is part of Chinese culture which has a long history dating back to 7,000 years ago when drinking was common in festivals and in business meetings, especially for men. The 2015 China Chronic Disease and Nutrition Surveillance (CCDNS) reported that the prevalence of current alcohol use (those who have drank in the last 12 months) among Chinese adults over 18 years was $41.3 \%$ overall, $61.7 \%$ in men, and $20.3 \%$ in women (5). In 2013, among the same population, the prevalence of current alcohol use was $37.1 \%$ overall, $58.3 \%$ in men, and $15.4 \%$ in women. The 2007 CCDNS reported the prevalence of current alcohol use in those aged 18-69 years was $35.7 \%$ overall, $55.6 \%$ in men, and $15.0 \%$ in women, which indicated a continuous increase (G).

\section{ALCOHOL POLICY IN CHINA}

In 2018, the World Health Organization (WHO) launched SAFER, a new initiative and technical package aimed to help governments to reduce the harmful use of alcohol. SAFER encompasses five highimpact strategies, namely: strengthening restrictions on alcohol availability; advancing and enforcing drunkdriving countermeasures; facilitating access to screening, brief interventions, and treatment; enforcing bans for comprehensive restrictions on alcohol advertising, sponsorship, and promotion; and raising prices on alcohol through excise taxes and other pricing policies. SAFER is a roadmap for governments to address the issue of harmful use of alcohol (7). In the following sections, alcohol policy in China were described according to the SAFER framework.

\section{Alcohol Availability}

Alcohol use is lawful in China, therefore the key to reducing alcohol consumption and alcohol-related harm is to control the accessibility of liquor. Since China adopted the Reform and Opening-Up Policy in the 1980s, the government discontinued the monopoly on alcohol (8). Different departments independently managed the materials, production, and sales of alcohol. Currently, China regulates alcohol under the Food Safety Law, in which the licensing system plays an important role. However, the decentralized management of alcohol production and circulation poses a major barrier for reducing the harmful use of alcohol because no sector can commit to taking full responsibility, and stakeholders have different interests and have difficulty reaching consensus. China has no enforceable legal provision to regulate when or where 
alcoholic beverages are sold. Setting the minimum age is an important measure to protect minors from alcohol consumption. The Law on the Protection of Minors bans selling alcohol to minors under the age of 18 years old (9) and the Health Promotion Law also explicitly prohibits the sale of alcohol to minors (10), but violations are commonplace. In China, several measures may be considered to control the availability of alcohol. A government monopoly can effectively limit the availability of alcohol. In China, where monopolies do not exist, harmonizing interests of all stakeholders and strengthening their cooperation can help effectively enforce licensing regulations. It is advisable to regulate the density of alcohol outlets and ban it where there is undue harm and to regulate the days and hours of sale. Moreover, strictly enforcing the ban on the sale of alcohol to minors plus limiting the secondary supply of alcohol from parents or friends can help this issue.

\section{Drinking and Driving Countermeasures}

Effective road safety transport policies are essential to reduce drinking and driving, which harms not only the drinker but also innocent people such as passengers and pedestrians. Drinking and driving has received special attention in China. The Road Traffic Safety Law of the People's Republic of China, revised in 2011, has been amended to impose harsher penalties. Based on blood alcohol concentration (BAC), the law imposed different punishments for drinking and driving $(20 \mathrm{mg} / 100 \mathrm{~mL} \leq \mathrm{BAC}<80 \mathrm{mg} / 100 \mathrm{~mL})$ and drunk driving (BAC $\geq 80 \mathrm{mg} / 100 \mathrm{~mL})$, including flat fines, penalty points, mandatory treatment, license suspension/revocation, imprisonment, short-term detention, and criminal penalty (11). Furthermore, drunk driving is listed in criminal law since 2011. An interrupted time-series analysis based on data from the Traffic Management Bureau of the Ministry of Public Security of the People's Republic of China showed that the average annual incidences of crashes, mortality, and injuries slightly dropped after the enactment of the amended laws, which is far from expectation (12). On October 30, 2020, the Traffic Management Bureau of the Ministry of Public Security organized the national public security traffic departments to carry out centralized and unified weekend night inspections, which investigated and punished more than 9,000 drinking and driving cases, including more than 1,500 drunk-driving cases (13). Drinking and driving remains an urgent problem in China, requiring effective enforcement of laws accompanied by strong public awareness campaigns.

\section{Screening, Brief Interventions, and Treatment}

Health services are central in helping individuals to tackle alcohol-related harms. Consistent evidence indicates that screening and brief interventions in healthcare settings could bring in a large health gain, although they are costly to implement. To date, China has not implemented systematic screening and brief interventions. Behavior and pharmacological therapies are effective in treating alcohol use disorders. In China, many patients suffering from alcohol-use disorders were untreated, although it ranked among the top 3 mental diseases. Several concrete steps were recommended to facilitate access to screening, brief interventions, and treatment by increasing the capacity of the health and social welfare systems by funding and supporting initiatives for screening and brief interventions and by enhancing availability, accessibility, and affordable health services especially to persons of low socioeconomic status.

\section{Restrictions on Alcohol Advertising, Sponsorship, and Promotion}

Alcohol advertising can recruit new drinkers and increase the amount of alcohol consumed by current drinkers, and it is therefore widely used by alcohol enterprises to promote sales. Bans or comprehensive restrictions on alcohol advertising, promotion, and sponsorship could help protect young people from alcohol marketing and reduce the reactivity of alcoholdependent persons. Currently, alcohol advertising is regulated under the Advertisement Law of the People's Republic of China (14) and the Regulation on Broadcasting of Radio and Television Advertisements (15). Legal provisions stipulate the content, form, quantity, time, and publishing media of alcohol advertisements, but these legal provisions are poorly enforced. In addition, there are no regulations on outdoor alcohol advertising and online alcohol advertising (16-17). China has several measures at its disposal to limit the marketing of alcohol by improving relevant laws and regulations, by developing effective systems of surveillance of marketing of alcohol products, and by establishing effective administrative and deterrence systems for violations.

\section{Raising Prices on Alcohol}

Raising alcohol prices leads to decreased alcohol 
consumption. Pricing and taxation were assessed to have the strongest impacts on alcohol consumption among all alcohol policy measures. Both a value-added tax (VAT) and excise taxes are imposed on alcohol in China. The VAT has been maintained at $17 \%$ (18), while the excise tax has been adjusted several times. The history of levying taxes in China is complicated and has been more focused on expanding production, sales, and resulting tax revenue instead of reducing harmful drinking. For example, grain wine was taxed at $50 \%$ in 1984 and decreased to $25 \%$ in 1995 and $20 \%$ in 2006, and it has remained at $20 \%$ following new policies in 2009 (11). Therefore, China's tax policy has a relatively low tax rate on alcohol that is not used as a tool to reduce harmful consumption. Raising the lowest prices on alcohol by introducing a legal minimum price per gram of alcohol has a potentially great impact on total consumption. Currently, there is no legal minimum prices for alcohol in China. China can try several steps to influence the final price of alcohol by increasing excise taxes, by establishing minimum prices for alcohol, and by restricting the use of price promotion.

\section{OPPORTUNITIES, CHALLENGES, AND THE WAY FORWARD}

Sustainable reaction to address the issue of harmful use of alcohol requires solid political will and commitment. In response to the huge burden caused by alcohol consumption, both the Healthy China 2030 plan (19) and Medium-to-Long Term Plan for the Prevention and Treatment of Chronic Disease (2017-2025) (20) (hereafter: "the two national plans") pointed out that the harmful use of alcohol should be reduced. This is the first time that China included the reduction of alcohol use in national level plans, which indicated that China has gradually attached importance to the harm of alcohol consumption. In addition, the government requires public officials to avoid extravagance and drinking high-end alcohol, which provides a strong role model effect in reducing alcohol consumption.

However, China's efforts to reduce harmful use of alcohol are still at the beginning. Although the two national-level plans pointed out the need to reduce harmful use of alcohol, neither gave specific targets for reducing alcohol consumption or drinking rates. China has not yet established a specialized agency responsible for reducing the harmful use of alcohol, let alone a mechanism for multisectoral coordination. In all five areas of SAFER which the WHO recommended, as mentioned above, China's current strategies have not yet been implemented or need to be promoted further.

Facing the huge public health challenge caused by harmful use of alcohol, the WHO proposed the Global Strategy to Reduce the Harmful Use of Alcohol in 2010 (21) and further launched the SAFER initiative, which are the most comprehensive documents with guidance on reducing the harmful use of alcohol. The harmful use of alcohol is closely relevant to the 2030 Agenda for Sustainable Development. As a member state, China should fully utilize the existing evidencebased cost-effectiveness strategies and measures recommended by the WHO and, based on the current situation of alcohol epidemiology, address the severe public health challenge caused by harmful use of alcohol.

doi: $10.46234 / \mathrm{ccdcw} 2021.012$

\# Corresponding author: Yamin Bai, baiyamin@ncncd.chinacdc.cn.

${ }^{1}$ National Center for Chronic and Noncommunicable Disease Control and Prevention, China CDC, Beijing, China.

Submitted: September 05, 2020; Accepted: December 31, 2020

\section{REFERENCES}

1. Rehm J, Gmel Sr GE, Gmel G, Hasan OSM, Imtiaz S, Popova S, et al. The relationship between different dimensions of alcohol use and the burden of disease-an update. Addiction 2017;112(6):968-1001. http://dx.doi.org/10.1111/add.13757.

2. World Health Organization. Global status report on alcohol and health 2018. https://apps.who.int/iris/bitstream/handle/10665/274603/9789 241565639-eng.pdf?ua=1. [2020-8-1].

3. Jiang YY, Liu SW, Ji N, Zeng XY, Liu YN, Zhang M, et al. Deaths attributable to alcohol use and its impact on life expectancy in China, 2013. Chin J Epidemiol 2018;39(1):27 - 31. http://dx.doi.org/10. 3760/cma.j.issn.0254-6450.2018.01.005. (In Chinese).

4. Institute for Health Metrics and Evaluation. GBD results tool results. http://ghdx.healthdata.org/gbd-results-tool/result/3793c9d78d4c8 27857ff7627ac55af8b. [2020-7-23].

5. Zhao ZP, Wang LM, Zhang M, Zhang X, Huang ZJ, Li C, et al. Preplanned studies: geographic distribution of alcohol use among Chinese adults-China, 2015. China CDC Wkly 2020;2(7):98 - 103. http://dx.doi.org/10.46234/ccdcw2020.029.

6. National Center for Chronic and Noncommunicable Disease Control and Prevention, Chinese Center for Disease Control and Prevention. Report on chronic disease risk factor surveillance in China 2013. Beijing: Military Medical Science Press. 2016. http://book.kongfz.com/ 277835/1109159365/. (In Chinese).

7. World Health Organization. WHO launches SAFER, a new alcohol control initiative. https://www.who.int/substance_abuse/safer/en/. [2020-8-3].

8. Guo X, Huang YG. The development of alcohol policy in contemporary China. J Food Drug Anal 2015;23(1):19-29. http://dx.doi.org/10.1016/j.jfda.2014.05.002.

9. The State Council of the People's Republic of China. Law on the Protection of Minors of the People's Republic of China. http://www. 
gov.cn/flfg/2006-12/29/content_554397.htm. [2020-8-3]. (In Chinese).

10. The State Council of the People's Republic of China. Basic Medical and Health Promotion Law of the People's Republic of China. http://www. gov.cn/xinwen/2019-12/29/content_5464861.htm. [2020-8-3]. (In Chinese).

11. The Central People's Government of the People's Republic of China. RoadTrafficSafetyLawofthePeople'sRepublicofChina.http://www.gov.cn/ ztzl/2006-05/27/content_292734.htm. [2020-8-3]. (In Chinese).

12. Fei GQ, Li XY, Sun QN, Qian YN, Stallones L, Xiang H, et al. Effectiveness of implementing the criminal administrative punishment law of drunk driving in China: an interrupted time series analysis, 2004-2017. Accid Anal Prev 2020;144:105670. http://dx.doi.org/ 10.1016/j.aap.2020.105670.

13. The Ministry of Public Security of the People's Republic of China. Police and traffic control departments carry out weekend night inspections all over the country. https://www.mps.gov.cn/n2254098/ n4904352/c7487271/content.html. [2020-11-3]. (In Chinese).

14. The National People's Congress of the People's Republic of China. Advertisement law of the People's Republic of China. http://www. gov.cn/guoqing/2020-12/24/content_5572939.htm. [2021-01-05]. (In Chinese).

15. National Radio and Television Administration. Order no. 61 of the national radio and television administration: Regulation on broadcasting of radio and television advertisements. http://www. nrta.gov.cn/art/2015/5/21/art_1588_43800.html. [2021-01-05]. (In
Chinese).

16. The State Administration for Industry and Commerce of the People's Republic of China. Order No. 25: regulations on the administration of outdoor advertisement registration. http://www.gov.cn/ziliao/flfg/200606/07/content_302493.htm. [2021-01-05]. (In Chinese).

17. The State Administration for Industry and Commerce of the People's Republic of China. Order No. 87: interim Regulation on the administration of Internet advertising. http://www.gov.cn/xinwen/ 2016-07/09/content_5089902.htm. [2021-01-05]. (In Chinese).

18. The State Council of the People's Republic of China. Order No. 538 of the State Council of the People's Republic of China: provisional Regulations of the people's republic of China on value added tax. http:// www.gov.cn/zwgk/2008-11/14/content_1149516.htm. [2020-8-3]. (In Chinese).

19. The Communist Party of China Central Committee and the State Council. "Healthy China 2030" Blueprint. 2016. http://www.gov.cn/ xinwen/2016-10/25/content_5124174.htm. [2020-8-3]. (In Chinese).

20. The State Council of the People's Republic of China. General Office of the State Council issued the medium-to long term plan for the prevention and treatment of chronic disease (2017-2025). http://www. gov.cn/zhengce/content/2017-02/14/content_5167886.htm. [2020-83]. (In Chinese).

21. World Health Organization. Global strategy to reduce the harmful use of alcohol. https://www.who.int/publications/i/item/9789241599931. [2020-8-3]. 